bereavement is due to a specific sort of disjunction - the loss of someone you love. After all, falling in love is also an abrupt disjunction, but it is often a lot of fun.

Given the range of adaptive problems that humans face (from both an evolutionary and development perspective), there is no reason to expect a single principle that governs interactions between the mind and the environment. In some domains, developmental malleability makes sense; in others, it does not. Sometimes adults should hate the new, when a loved one dies, for example; sometimes we should embrace it, such as when starting a promising relationship. The relationship between the mind and the environment is too complex for a one-line theory.

Paul Bloom is in the Depar tment of Psychology,

Yale University, New Haven,

Connecticut 06520-8205, USA.

\title{
A turbulent history
}

\section{Worlds of Flow: A History of \\ Hydrodynamics from the Bernoullis \\ to Prandt। \\ by Olivier Darrigol \\ Oxford University Press: 2005. 376 pp. £35, $\$ 74.50$}

\section{Roddam Narasimha}

The continuing fascination of hydrodynamics - or its modern, more inclusive offspring fluid dynamics - is due to the fact that many phenomena (such as turbulent flows) that we can observe with our unaided senses pose deep scientific problems that have not been solved to this day. Those unaided observations have led artists and scientists to wonder at the beauty, majesty and waywardness of flows over the centuries. Leonardo da Vinci's pictures of vortices, Hokusai's prints of waves, and the unknown Sanskrit poet's celebration of the splendid diversity of flowing water in current, wave, foam and spray - all these are matched by the scientist's struggle to understand flow and the engineer's attempts to manage it.
The governing equations of hydrodynamics were first written down by the French engineer Claude Louis Navier in 1822. Those equations (with which the name of George Stokes is also associated) remain valid, so the fact that turbulence, for example, has resisted a final solution must be attributed to the inadequacy of our mathematics to handle the strong nonlinearity of the equations and the almost universal tendency of flows to crumple into one form of instability or other except under the mildest conditions. John von Neumann saw the problem clearly when he said that ${ }^{\text {c }}$ The impact of an adequate theory of turbulence on certain very important parts of pure mathematics may be even greater" (than on fluid dynamics). The basic mathematical nature of the problem is now being more widely recognized: one of the seven million-dollar 'Millennium Prize' problems identified by the Clay Mathematics Institute in Cambridge, Massachusetts, has to do with Navier-Stokes solutions. Understandably, it is this very inadequacy of the mathematics that has made physical insight, clever experiments and smart approximations such prized virtues in fluid dynamical research.

Until now, anyone interested in the history of this subject has usually had to turn to books such as History of Hydraulics (Institute of Hydraulic Research, 1957) by Rouse Hunter and Simon Ince or to John Anderson's A History of Aerodynamics (Cambridge University Press, 1997), both strongly oriented to specific engineering disciplines, and a few other rather specialized works. Oliver Darrigol's Worlds of Flow is the first book to see hydrodynamics in the wider context of the history of ideas in science. The subject has finally found the distinguished historian it deserves, and the serious history it demands.

Darrigol approaches the subject through the evolution of the concepts that now describe the motion of fluids - viscosity, vorticity, waves, instability, turbulence. He begins with what he calls the small elite of eighteenth-century Swiss and French 'geometers' (including Daniel and Johann Bernoulli), and progresses to the engineers, mathematicians and physicists of the 19th century. During this time, the subject was divided into the ideal world of the hydrodynamicist, who did beautiful mathematics that often failed reality checks, and the real world of the 'hydraulician' who collected useful formulas disconnected from dynamics. These two 'worlds' of flow evolved separately, generally with scorn for each other.

But around the end of the nineteenth century and into the twentieth, many engineers began to examine the foundations of the subject in their own rather pragmatic ways: Osborne Reynolds's studies of turbulent flow, William Rankine's analysis of shock waves and Ludwig Prandtl's many distinctive theories came to characterize an emerging 'engineering

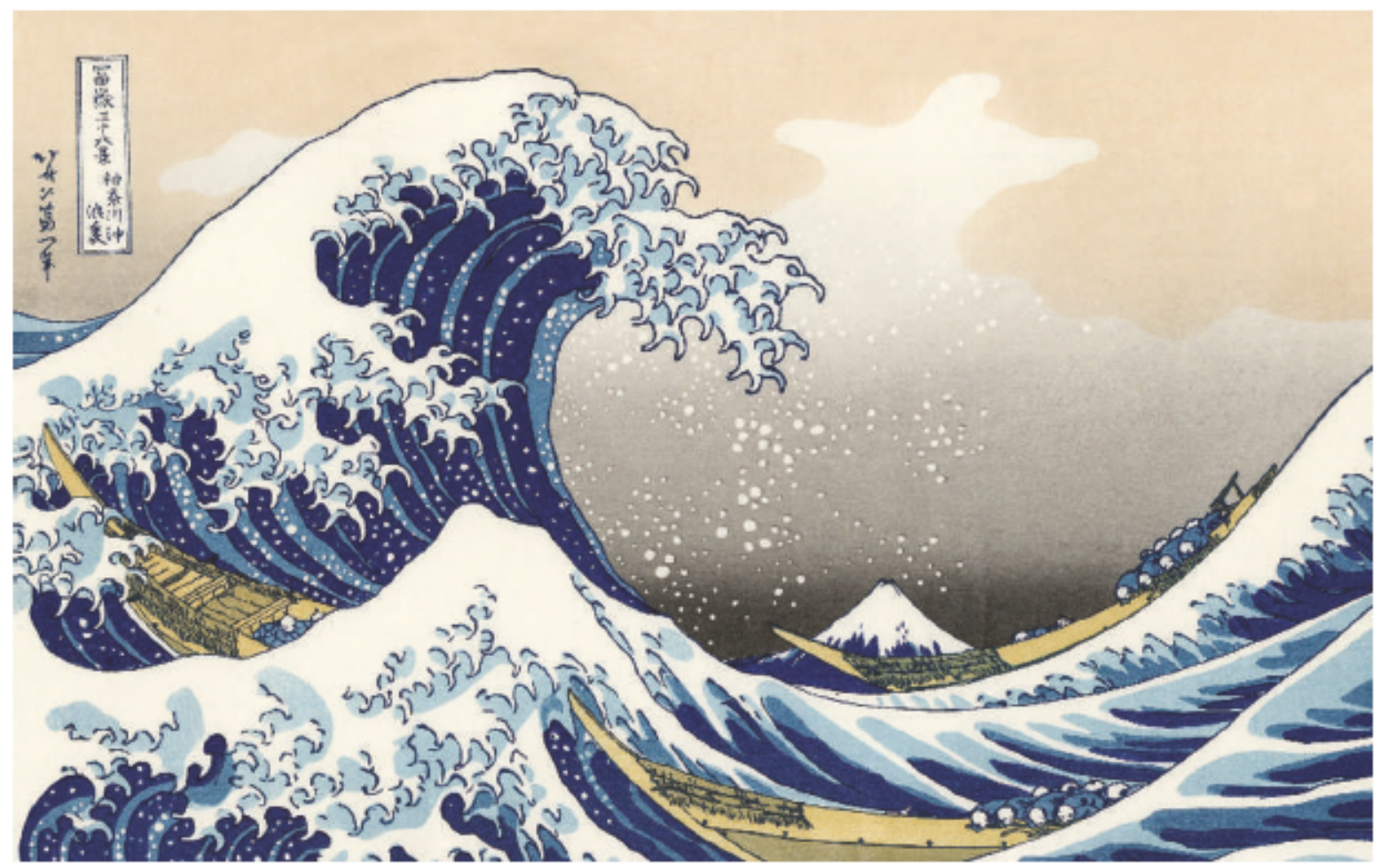

Fluid power:

The Great Wave by the seventeenthcentury Japanese artist Hokusai. 
science. All of these were inspired by the earlier work of physicists and mathematicians, and often appealed to the Navier-Stokes equations. But they also introduced novel approximations and did not hesitate to use purely numerical methods when no analytical solutions were available. Meanwhile, the hydrodynamicists discovered that the real-life problems that the engineers were tackling involved deep questions in physics and mathematics. The study of the instability of fluid motions - the subject of Darrigol's fifth chapter - exemplifies these developments best: the two cultures began to meet, as the formulations of William Orr, Arnold Sommerfeld and Lord Rayleigh led both to the striking work of G. I. Taylor on cylindrical Couette flow and Werner Heisenberg on plane Poiseuille flow, and to the prescient work on boundary layers by Prandtl and Walter Tollmien.

Darrigol handles these developments with scholarship, insight and charm. One of the fascinating episodes he describes is the vortex theory of matter. For William Thomson (later Lord Kelvin) the idea that matter was made up of vortex rings had an extraordinary appeal: but the theory needed vortex rings to be stable, which unfortunately they were not. It seems to me that it was such failures, amid the striking success of James Maxwell's electromagnetic theory and other exciting developments in relativity and quantum mechanics, that were largely responsible for physicists' fading interest in fluid dynamics. It was thus largely left to the applied mathematicians and the engineers to fashion whatever successes the twentieth century could claim.

Darrigol takes us through these developments in the still incomplete history of the subject with a rare balance that accurately reflects its rich complexity (although I did miss mention of V. W. Ekman's friction layer in the rotating ocean and L. F. Richardson's heroic failure at numerical weather prediction). This is a book that all practising fluid dynamicists must read: I hope there will be a paperback edition soon, so that the strange history of the subject that Darrigol describes with such insight will become part of the intellectual legacy of interested students in engineering, mathematics and physics.

Roddam Narasimha is at the Jawaharlal

Nehru Centre for Advanced Scientific

Research, Jakkur, Bangalore 560 064, India.

\section{Form and flow}

\section{A sculptural approach to the heart.}

Philip J. Kilner

During my medical training and early years as a doctor, I was repeatedly drawn to art, even if itseemed to me then to lack relevance to medical work. Art felt liberating and satisfying, but subjective and impractical, whereas the kind of scientific thought that I wascoming to adopt seemed convincing and applicable, yet strangely out of touch with human feelings or values. I left medicine for several years and studied sculpture at Emerson College inSussex, England. As Martin Kemp has pointed out, science and art are nothomogeneous categories (Nature 434, 308-309; 2005). What I found atEmersonCollege was an observational approach tonatural science, and anartistic approach that was nourished by natural forms and processes without necessarily being representational. This was a rewarding mix, fostering discovery, creativity and a sense of wonder. The receptive, phenomenological approach we used owed much to Johann Wolfgang von Goethe (1749-1832), who regarded his scientific studies as more significant than his literary work.

Like Goethe, we made observations of form and transformation in plants, animal bones and the human skeleton. Wewere taught by John Wilkes, the originator of a range of elegantly sculpted surfaces called Flowforms, shaped to induce rhythmically alternating vortices inwater streaming over them

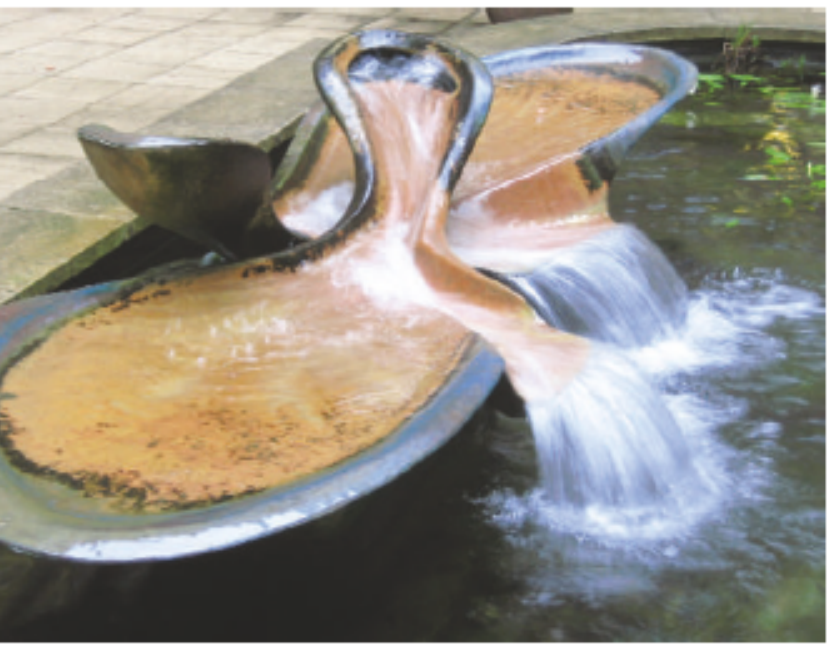

related to congenital heart disease and heart surgery.

But was art really significant as part of this mix, or was it just the practical approach that provided a basis for research on flow through the heart? The directengagement with three-dimensional form and flow was relevant, but other aspects of the artistic approach wereequally important.

One of these is the relative freedom to explore. Each artistic field offersits own playground for exploring the associations between phenomena. And the arts, traditionally at least, imply

(www.virbelaflowforms.anth.org.uk).We learned to guide the movements of water and shape the swirling, fluctuating vortices, gradually gaining insightinto the morphodynamics of flow. Creative engagement rarely resulted in a product that stood the test of time, but regularly led to fresh insight and discovery. I remember imagining and then shaping a variant of Wilkes' Flowform designto makea simple, asymmetric cavity that could convert continuous inflow to pulsatile outflow through a spontaneouscycle of accumulation and discharge. A pair of such forms (see above) can be seen at Royal Brompton Hospital in London.

Over the next 20 years, this discovery led me to explore the forms and dynamics of flow through heart cavities (see Nature 404, 759-761; 2000), to a career in cardiovascular imaging, and to research aesthetic appreciation. Beauty and, perhaps, meaning tend to be apparent not through analysis but throughinclusive, intuitive perception, which, like analytical and theoretical approaches, requires practice and training. Artistic exploration, whether among forms, colours or sounds, fosters a more flexible, inclusive awareness than analysis. If that kind of embracing awareness were adequately represented in scientific institutions as a counterpart to the analytical, I might feel less need towrite this. But as far as I have exerienced, the culture of science favours analysis at the expense of contextual awareness. The latter is at least as important, particularly whendealing with the organic, theernironmental, and the human. Philip J. Kilner is a consultant and reader in cardiovascular magnetic resonance at Royal Brompton Hospital and Imperial College, London, UK. 